\title{
THE TREATMENT OF WOUNDS OF BLOOD VESSELS.
}

\section{By Colonel PHILIP H. MITCHINER, C.B.E., M.D., M.S., F.R.C.S.}

(Surgeon to St. Thomas's Hospital.)

Wounds of blood vessels are a natural sequence of damage to any body tissue and the treatment of such vascular lesions is therefore of considerable interest and importance. It cannot be too strongly emphasised that the primary consideration in the treatment of blood vessel wounds is hæmostasis and this must be thorough and absolute, as without it the danger of wound infection and subsequent grave danger to the patient's life are inevitable. In addition steps must be taken to conserve if possible the vitality of the part supplied by the damaged vessel and for this reason any operation which is designed to conserve the flow of blood through the damaged trunk at any rate until the collateral circulation is established is worthy of consideration.

\section{First Aid.}

It must be realised that First Aid Treatment is essentially life saving in cases of vascular lesions and must be prompt and efficient; such treatment consists, in most cases, in the application of local pressure to the wound by means of a surgically sterile dressing and this usually suffices to stop the bleeding. In case of damage to large arteries or where no sterile dressing is available, it is best to apply some form of tourniquet to the limb immediately above the wound, care being taken that this is applied only just so tightly as to arrest the bleeding, that the time and fact of application are recorded on the patient's forehead, and that the tourniquet is loosened every 20 minutes.

\section{Further Treatment.}

When the case reaches hospital the wound must be treated by excision in the operating theatre with the patient anæsthetised. The blood vessels must not be picked up in a haphazard manner together with a mass of crushed tissue through which they are oozing, therein lies grave danger of gross infection; all such damaged tissue must be excised till spurting vessels are met and these picked up cleanly and ligatured. In the case of small vessels of all sorts, crushed veins, and completely severed arteries this is all that can be done both to arrest hæmorrhage and as local treatment to the vessel.

Care must be taken after clearing all clot from the hæmatoma in the tissues to secure proper coaptation of the deep tissues and if this is not possible without tension the wound must be left open and subsequently closed in a few days, when it is clear no infection is present, by secondary suture. The wound should be lightly dressed and firmly bandaged. The general circulation of the part should be maintained by elevation and rest secured by suitable splintage, preferably of the skeletal variety with extension as this enables both the state of the wound and the condition of the circulation in the peripheral tissues to be observed, but the ring of the splint must in no way restrict the circulation of the limb.

It is then, only in those cases where contusion or partial division of large arteries have occurred, or where a small penetrating wound involves contiguous artery and vein, that reparative surgery has any place in these cases of wounds of blood vessels.

Even in these cases it is often wise to ligature both artery and vein above and below the lesion and divide them completely across, for as Makins shewed in the 
late war this " simultaneous" ligature of both main artery and vein is less likely to be followed by gangrene of the limb than if one or other vessel alone is ligated. Furthermore the results of reparative surgery on blood vessels in war conditions is notoriously disappointing, fully two-thirds of such cases thrombosing locally, while in the remainder there is considerable tendency for the repaired vessel wall to yield subsequently with the formation of another aneurysmal dilation. Moreover, it may be questionable in the rush of a casualty hospital if it is justifiable to take considerable time over an operation on one subject the results of which are considerably in doubt while many other urgent cases call for prompt attention from the surgeon. On these grounds therefore it will be seen that the application of reparative surgery in wounds of even the large blood vessels has a very limited field; on the whole the results of repair of veins are more successful than those of arteries where thrombosis is far more prone to follow local surgical procedures.

\section{Local Repair Operations.}

In cases where local repair is undertaken temporary arrest of the vascular flow is essential and is best secured by temporary ligature of the vessels with a fine sterile tape or the application of some form of light tourniquet forceps, rather than by the application of a tourniquet to the limb as a whole.

Few special instruments are needed beyond the tourniquet forceps, but fine thread (I90 size) which has been well soaked in sterile paraffin or white vaseline by heating in these media is essential and needles must be very fine and round bodied, while the handling of tissues, especially the damaged vessels must be very gentle and further reduced to a minimum. It is claimed that the drip infusion of heparin solution for some twenty-four hours after operation goes far to reduce the risk of local post-operative thrombosis, but such a procedure is not without risk, especially in the case of a shocked and debilitated war wounded patient.

\section{Types of Vessel Wounds.}

In arterial wounds three conditions may be met with:-

(1) Contusion.

(2) Severance of the adventitia and media with the intima bulging.

(3) Complete division of all coats of the vessel.

(I) In cases of contusion the artery is red and hard with absence of pulse both at the site of lesion and distally to it. Inasmuch as the vessel wall is crushed and bruised local removal of clot is likely to be followed only by further clotting and personally we do not advocate local surgery in these cases, except to ligature the artery above and below the lesion and excise the damaged part. This is advisable as if left the subsequent fibrosis causes tension in the course of the vessel and often leads to considerable vascular disturbance, pain and claudication in the limb beyond. Those who advocate local removal of clot, usually make a longitudinal incision in the vessel above the contusion and suck the clot out, subsequently sewing up the incision in the wall with vaselined thread; it is not wise under any conditions to incise the contused area of the vessel.

(2) In cases where the intima is bulging through a torn media, the adjacent vein is often lacerated or bruised and if no local surgery is performed this type of injury results ultimately in the formation of an arterio-venous aneurysm. Surgery must therefore be undertaken when the wound is excised; this had best take the form of the suture over the herniated intima of a piece of tissue taken from 
the sheath of the vessel, as attempts to sew up the media are not very successful, the stitches tearing out when the blood is readmitted to the arterial trunk, excepting in the case of longitudinal tears, which are, in our experience, seldom met with. If the vein is lacerated the wound if small should be sutured, when the edges have been trimmed, but if extensive it is best to ligature the vein above and below and remove the damaged segment.

(3) Division of all coats of the vessel if these are much crushed calls for ligature of the vessel above and below the lesion and excision of the damaged part of the arterial wall; similarly if more than half the lumen is divided transversely across ligature should be practised as the scar in most cases yields subsequently with the formation of an aneurysm, even if thrombosis does not result immediately after the operation, which is generally the case. It is, only, then the case of longitudinal cuts and transverse ones involving less than half of the vessel wall that local repair can be undertaken with assurance of success, and even then only if the vessel wall is not crushed or lacerated.

\section{Operation.}

In such cases, temporary arrest of the blood stream having been secured, the vessel wall is cleaned by saline washing from all clot, and then sutured with fine greased thread; it is essential that intima be approximated closely to intima

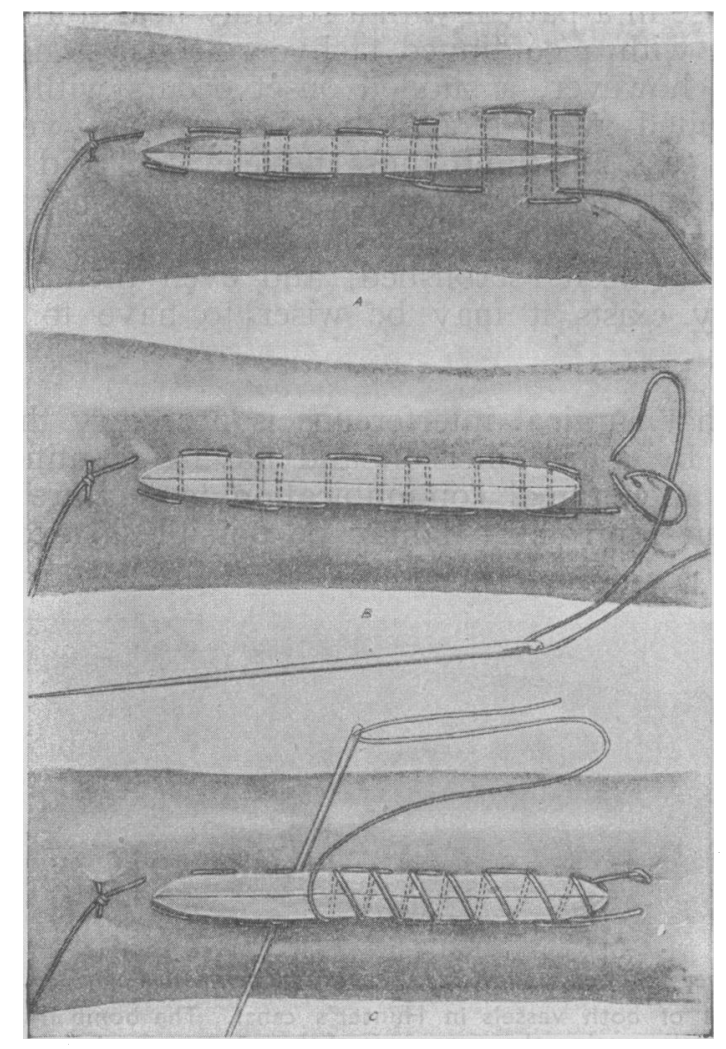

Dorrance's method of suture of a longitudinal wound of an artery. (From Romanis \& Mitchiner's Surgery-Reprint by permission of J. \& A. Churchill.) 
at the suture line or extensive thrombosis will occur, while as little stitching as possible should shew in the lumen of the reconstructed vessel. To secure these ends it is well to evert the edges of the wound by the sutures, care being taken that in so doing the lumen of the artery is not narrowed unduly. Subsequently the wound in the soft tissues will be closed without undue tension and the part elevated and splinted to ensure rest. If much blood has been lost or the patient is greatly shocked, then intravenous drip glucose-saline or blood transfusion must be administered and to this heparin may be added. Splintage will be maintained for at least fourteen days, after which gentle movements of the limb are to be encouraged with the patient prone in bed; no getting up or more active use must be permitted for a month from the time of operation.

Wounds of veins are treated on the same lines, while the vessel wall and lower blood pressure make operation far easier; thrombosis is more likely to occur at the suture line though not so prone to occlude the entire vessel lumen as in the case of the arteries. Success is therefore easier to obtain though not so essential to the life of the limb where the re-establishment of arterial flow for a few hours may enable the peripheral circulation to re-establish through collateral vessels and so avert gangrene beyond the site of the wound; this is especially the case in the lower limb and with patients above forty-five years of age.

Arterio-venous communications usually develop gradually following injuries involving main trunks which lie contiguous and are indicated by the persistence of an unduly rapid pulse in a patient with a soundly healed and uninfected wound. If infection be present with a continued tachycardia gas gangrene should be suspected. Occasionally, however, a sudden onset occurs with severe collapse and a limb greatly distended with blood shewing marked venous distusion and pulsation; in such a case the limb must be elevated and bandaged from the periphery and the patient treated for shock. It is wise never to interfere surgically in any case of arterio-venous aneurysm until it is certain that an adequate collateral circulation has been established, and even then in some cases where no pain and disability exists it may be wiser to have to resort to an elastic supporting bandage.

If it is decided that surgical interference is necessary then the best method is to expose the area by a free incision and having identified the main vessels above and below the aneurysmal communication to ligature these and ascertain that pulsation has been controlled; it may be possible to excise the sac and this

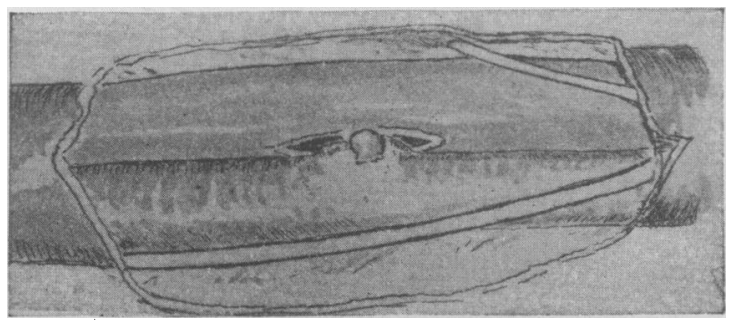

(From Mitchiner \& Cowell. Air-Raids. Reprint by permission of J. \& A. Churchill.) Gunshot wound of both vessels in Hunter's canal. The bomb fragment passed between the vessels, cutting the outer coat of the artery and opening the vein. At the operation the vein was sutured and the artery repaired by sewing a flap cut from the roof of the canal round the bulging inner coats. Result : Perfect recovery. 
should be done if it can be carried out without undue risk of damage to surrounding anatomical structures which will probably be adherent in the vessel walls and scar tissue. It may rarely be feasible to dissect out and close the communication between the vessels after temporary ligature to arrest the blood stream, but in most cases this is neither feasible nor does it give such satisfactory results as ligation of the main vessels above and below with excision of the sac.

\section{Traumatic Aneurysms.}

Similarly in late traumatic aneurysms seen on smaller arteries after stab wounds and occasionally subcutaneous contusions, ligature of the vessel above and below with complete extirpation of the sac is to be aimed at; no attempted repair operation ever gives satisfactory results in these cases.

\section{SYNOPSIS.}

It will thus be seen that in wounds of vessels the first aim is adequate hæmostasis and that this in the majority of cases means ligature of both ends of the vessel and its complete division to prevent subsequent vascular troubles from tension of scar tissue; there are, however, certain well defined injuries where local repair is justifiable and may be carried out with hope of success even in the adverse conditions of war surgery. 